\title{
High Speed Oblique CT System for Solder Bump Inspection
}

\author{
Atsushi TERAMOTO*, Muneo YAMADA*, Takayuki MURAKOSHI *, Masatoshi TSUZAKA**, and Hiroshi FUJITA *** \\ *Nagoya Electric Works Co., Ltd. \\ **Department of Radiological Technology, Nagoya University \\ ***Department of Intelligent Image Information, Graduate School of Medicine, Gifu University \\ ( tera@oe.nagoya-denki.co.jp )
}

\begin{abstract}
The high-density LSI packages such as BGA (ball grid array) and CSP (chip scale package) are widely used in the electrical products. Solder bump is used as an electrical junction of these packages, and they cannot be observed from outside. $X$ ray fluoroscopy is used as inspection method. It cannot evaluate the shape of solder bump correctly because information is compressed along the ray. The oblique $C T$ is considered as a method for accurately examining the shape of the solder bump. Conventional oblique CT still has the speed and mechanical constraint, and it cannot be introduced into the soldering line. In this paper, we propose the novel oblique CT system that can nondestructively obtain the 3D shape of solder bump at a high-speed. This system obtains the projection images from various directions using the rotational transfer of flat panel detector and the fixed mounted open-type $X$-ray generator that has a wide radiation angle, and reconstructs 3D image using the 3D FBP (Filtered Back Projection) method. In addition, we have developed the reconstruction acceleration unit for oblique $\mathrm{CT}$, and thus the $3 \mathrm{D}$ image is obtained in the real time. In our experiments, we evaluated the solder joints of BGA mounted substrate. The result demonstrated that the proposed system obtained the information that was able to determine the good or defective situation in soldering. This system is the world's first CT system, which can be introduced into the soldering line, and it is operated by more than 20 domestic and foreign factories now.
\end{abstract}

\section{INTRODUCTION}

Owing to the requirement of thin and light-weight products, BGA (ball grid array) and CSP (chip scale package)[1,2] have been widely used in the PCB (printed circuit board). The electrical junction of the BGA and CSP is formed by solder bumps that are placed in a grid-like formation under the LSI package. Though BGA and CSP brought the densifying the $\mathrm{PCB}$, inspection of soldering becomes very difficult because the solder junction hides from the view and optical inspection method cannot be applied. In the non-destructive inspection of the internal conditions such as the solder junction of the BGA and CSP, X-ray inspection is suitable. Since the advent of BGA and CSP, X-ray inspection has been widely used in the soldering line.

With regard to the inspection technique using X-ray fluoroscopy, the authors have developed techniques for extracting the solder using the energy subtraction method [3], quantitatively measuring the small solder bump at high speed using the relation between brightness and object thickness in the X-ray image [4], and detecting microvoids (bubbles) included in a small solder bump by using image data processing and statistical methods [5]. The inspection using Xray fluoroscopy is suitable for the fast inspection of consumer products because it employs simple mechanics and is able to obtain 2D information from the detector at a time. The X-ray fluoroscopic image, however, is the projection image, and the information in it is compressed along the $\mathrm{z}$ direction. Thus, it is impossible to evaluate the 3D solder shape correctly.

Recently, BGA and CSP are being applied to car electronics and communications infrastructure products which high reliability is required. In order to meet the quality requirement for these products, the establishment of the inspection technique that can examine solder bump more directly is strongly required. In addition, the inspection of the entire shipped product is preferable to guarantee its reliability. To achive the complete inspection, rapidity and mechanism assuming the use in the soldering line are required.

$\mathrm{X}$-ray CT (Computed Tomography) is a precise technique used for inspecting solder bumps [6,7]. Several industries use CT to obtain projection data by rotating the sample about an axis that is orthogonal to the axis that connects the X-ray generator to the detector. We call this method vertical CT in this paper. When the vertical CT inspects thin and wide objects such as PCBs, it is necessary to cut out the region of interest of the PCB because it touches the X-ray generator or detector during the scanning process. Since this technique is incapable of achieving non-destructive inspection, it is not used for inspection before a product is shipped but is used for defect analysis during research and development.

As another CT technique, oblique CT (OCT) was proposed [8-10]. It obtains the projection image of an object from an oblique direction by rotating the X-ray generator and detector. And some systems employed rotation stage and X-ray generator and detector are tilted for the rotational stage. In the OCT, scanning objects move horizontally because X-ray radiation is inclined; thin and wide objects such as PCBs do not touch the X-ray generator and detector. Therefore, although the PCB may be close to the X-ray generator, we can obtain a $3 \mathrm{D}$ image with high magnification without destroying the PCB. 
Conventional OCT is, however, proposed for medical use. When we introduce OCT into soldering line, there are three issues. Firstly, it is not possible to meet the speed requirement of soldering line because mechanical load (rotation mechanism in X-ray generator, stage and detector) is very heavy. Secondly is the visual field size. The projection images are collected by imaging tube as a $0.3 \mathrm{M}$ pixel in the conventional system. The visual field size in OCT image is very small for the size of the entire component under the necessary magnification. Consequently there are large gap between inspection time and production cycle in the conventional system. Thirdly is a mechanical adaptation to production process. Although inspection system that is introduced into soldering line requires the PCB transfer system, it is very difficult to introduce it to the conventional system by structural constraint.

In this paper, we propose the novel oblique CT system that can non-destructively obtain the $3 \mathrm{D}$ shape of solder bump at a high-speed. This paper is organized as follows. In Sec.II, a description of the OCT structure and processing procedure is given. In Sec.III, we show some experimental results. The conclusions are provided in Sec.IV.

\section{SYSTEM DESCRIPTION}

\section{A. Structure and processing outline of OCT}

The structure of the proposed system and the photograph of a prototype system are shown in Fig.1 and Fig.2, respectively. The system consists of flat panel detector (FPD, Fig.3(a)) with rotational transfer mechanism, the $\mathrm{X}-\mathrm{Y}$ stage, and an open-type $\mathrm{X}$-ray generator (Fig.3(b)) that is set on the elevator.

By synchronizing the rotational transfer of FPD and the X-Y movement of sample, the $X$-ray projection images from various directions are obtained. Then images are transferred to the host memory via image grabber board, and the 3D OCT image is obtained by executing $3 \mathrm{D}$ reconstruction on the reconstruction acceleration unit.

In this system, X-ray generator needs not move during the scanning because the radiation angle of open-type X-ray generator is 130 degrees. FPD which have to move during scanning is lightweight, it results in a low mechanical load; this further allows high-speed scanning.

Regarding to the number of pixels in the projection image, FPD has 5.3M pixel, it is able to obtain the 17 times area for the conventional imaging tube $(0.3 \mathrm{M}$ pixel).

Projection images are captured by geometrically magnified in order to obtain the solder shape in detail. Geometric magnification depends on the position of the PCB between the $\mathrm{X}$-ray generator and FPD on the relationship of the focus-todetector distance (FDD) and focus-to-object distance (FOD). Magnification equals FDD divided by FOD. As described in the previous section, the X-ray generator and the detector do not interfere when the direction of the X-ray irradiation is inclined. Therefore X-ray generator is able to approximate to the PCB, OCT image under high geometric magnification is obtained. The maximum magnification of $200 \times$ can be achieved in our prototype with $\mathrm{FDD}=300$ and $\mathrm{FOD}=1.5 \mathrm{~mm}$.
The spatial resolution equals pixel pitch of FPD divided magnification, finest resolution is $0.25 \mathrm{um}$.

With regard to the PCB handling, it is automatically inserted and exposured by the conveyor that equipped on the $\mathrm{X}-\mathrm{Y}$ stage, and the on-line inspection is feasible.

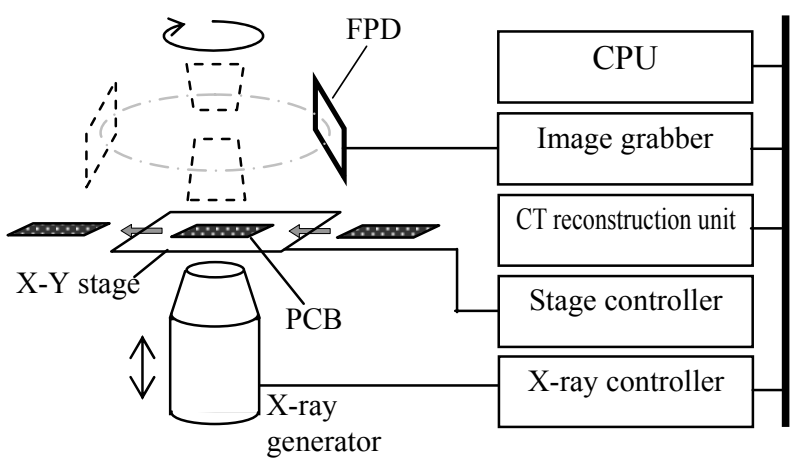

Fig.1 Schematic layout of OCT system. The system consists of a rotational $\mathrm{FPD}$, an $\mathrm{X}-\mathrm{Y}$ stage and an open-type X-ray generator. The FPD and the sample rotate simultaneously, and $\mathrm{X}$-ray projection images are obtained from various directions. The X-ray projection images are transferred to the host memory via an image grabber board, and 3D OCT image is obtained using reconstruction acceleration unit.

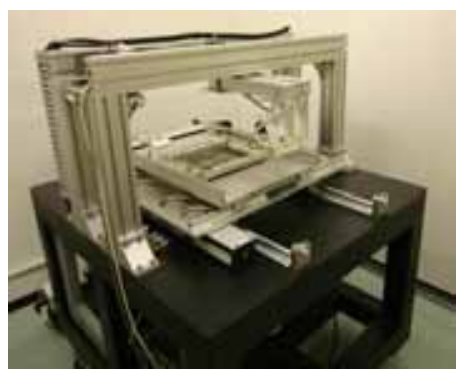

Fig.2 Prototype of OCT

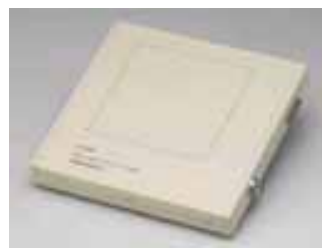

(a)

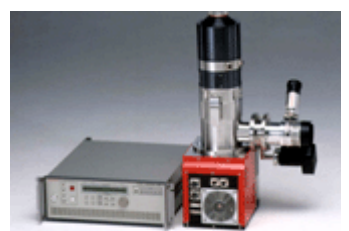

(b)
Fig.3 Key components of the OCT such as flat panel detector (a) and X-ray generator (b) . 


\section{B. Data processing}

The data processing procedure in the OCT consists of (1) collection of the projection image, (2) image correction, and (3) 3D image reconstruction. They are described as follows.

\section{(1) Collection of the projection images}

By synchronizing the rotation of FPD and the X-Y movement of sample, the X-ray projection images from various directions are obtained. Here, the center of projection image must keep noticing the identical position while collecting the projection images. The $\mathrm{X}-\mathrm{Y}$ stage is controlled so that identical position may rotate in radius of gyration $\mathrm{RR}$ synchronizing to the FPD as described in Fig.4, and gyration $R R$ is defined as follows.

$$
R R=F O D \cdot \tan \alpha
$$

where the radiation angle of the X-ray focus spot and the detector is $\alpha$.

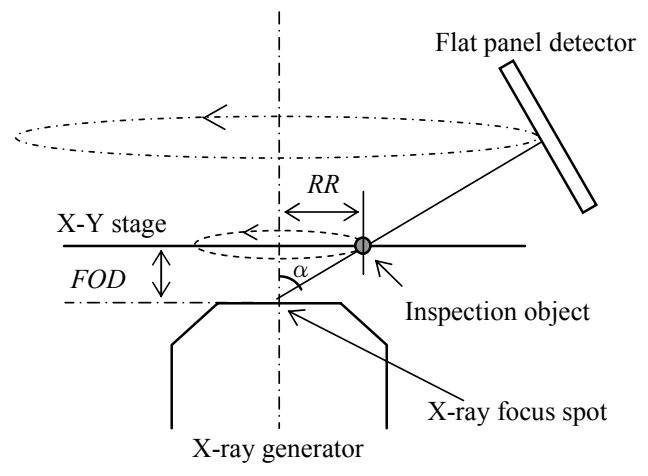

Fig.4 Synchronization of X-Y stage and FPD. The X-Y stage is controlled so that identical position may rotate in radius of gyration RR synchronizing to the FPD

\section{(2) Image correction}

The projection images have spatial non-uniformity in the proposed system. A non-uniformity corrected projection image $p(m, n)$ is calculated as follows:

$$
p(m, n)=-\log _{e} \frac{I(m, n)}{I_{0}(m, n)}
$$

where $I(m, n)$ is the X-ray intensity of projection, and $I_{0}(m, n)$ is the X-ray intensity without a sample.

\section{(3) 3D image reconstruction}

Fig.5 shows a simplified scheme of the OCT that is equivalent to the scheme in Fig.1. An object $f(\vec{r})$ is illuminated by a cone beam X-ray at the rotation angle $\phi$. We assume that $\vec{a}$ and $\vec{b}$ are unit vectors along the horizontal and vertical directions of the detector plane in the $3 \mathrm{D}$ space, and $\vec{c}$ is the vector that indicates the direction of projection. The 2D projection $p_{\phi}(m, n)$ of the object $f(\vec{r})$ is denoted as follows:

$$
p_{\phi}(m, n)=\int_{-\infty}^{\infty} f(\vec{r}) d l
$$

where $\vec{r}$ is defined as $\vec{r}=l \vec{c}+m \vec{a}+n \vec{b}$.

Provided that the X-ray projection is a parallel beam, the $2 \mathrm{D}$ Fourier transform of the projection image, $P_{\phi}(u, v)$, is equal to a vertical section of $\vec{c}$ in the 3D Fourier image $F(\vec{\omega})$ based on the projection slice theorem.

$$
F(u \vec{a}+v \vec{b})=P_{\phi}(u, v)
$$

The major methods of image reconstruction from projections are the Fourier method and FPB (Filtered Back Projection) method. In this study, we employed FBP to allow high-speed reconstruction of $3 \mathrm{D}$ images. $3 \mathrm{D}$ image reconstruction using FPB is realized by performing filtering and back projection. In the former, a frequency filter $H(u, v)$ is applied to the projection image $P_{\phi}(u, v)$, as shown in Eq.(5). In the latter, the filtered image $g_{\phi}(m, n)$ is back projected to the $3 \mathrm{D}$ space, as shown in Eq. (6).

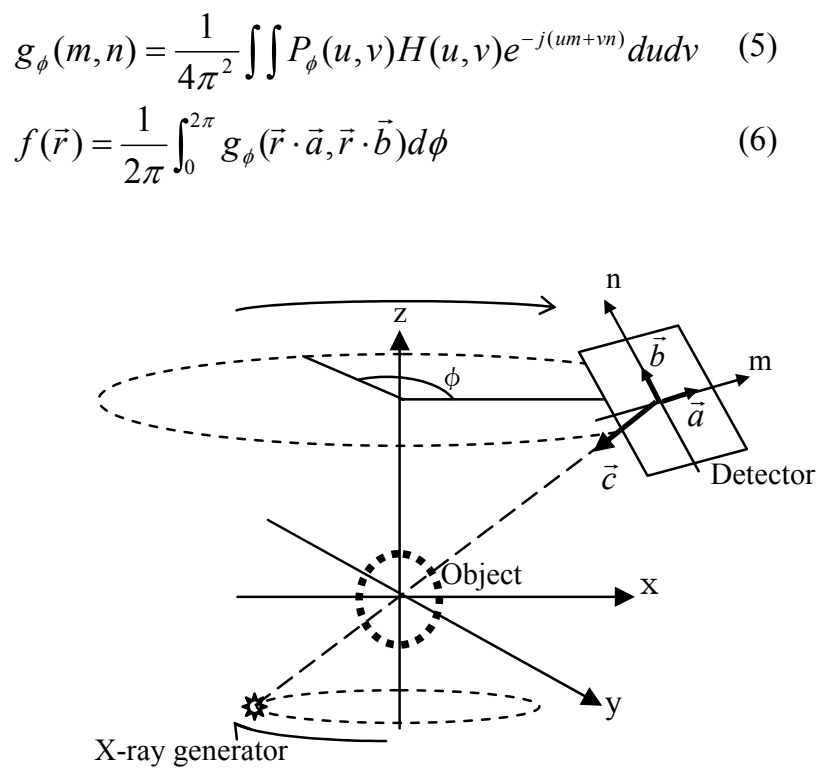

Fig.5 Simplified scheme of OCT. An object is illuminated by a cone beam X-ray at the rotation angle $\phi$. We assume that $\vec{a}$ and $\vec{b}$ are unit vectors along the horizontal and vertical directions of the detector plane in the $3 \mathrm{D}$ space, and $\vec{c}$ is the vector that indicates the direction of projection.

\section{Acceleration of $3 D$ image reconstruction}

In the OCT system, filter processing of the projection images followed by back projection to the 3D space are performed, as described in the previous section. The matrix size depends on the memory buffer size of computer; the maximum size of our prototype system was $2048 \times 2048 \times 32$ at 32 bits per pixel or 
$2048 \times 2048 \times 64$ at 16 bits. The number of calculations reaches four billion per projection excluding the memory access. Moreover, a large number of random accesses to the 3D memory buffer occur during the back projection processing.

We implemented the reconstruction software on the Windows platform. The processing time was $4.0 \mathrm{~s}$ per projection using a dual Xeon $3.6 \mathrm{GHz}$ processor with $4 \mathrm{~GB}$ RAM. However, the image acquisition time including a mechanical movement is $1 \mathrm{~s}$ per projection; the reconstruction is not calculated in real time. To accelerate the reconstruction speed, we developed a $\mathrm{CT}$ reconstruction acceleration unit for OCT. Fig.6 shows the block diagram of the CT reconstruction acceleration unit. It consists of three FPGAs (field programmable gate arrays) with a fast cache memory connected to each of them, and the projection and 3D OCT images are transferred over the PCI-X slot. The reconstruction time is $0.3 \mathrm{~s}$ per projection using this unit, which is 13 times faster than CPU processing.

The acquisition of the projection image and the reconstruction process can be executed in parallel, and the acquisition time is longer than the reconstruction time. Therefore, we can obtain the 3D OCT image in real time.

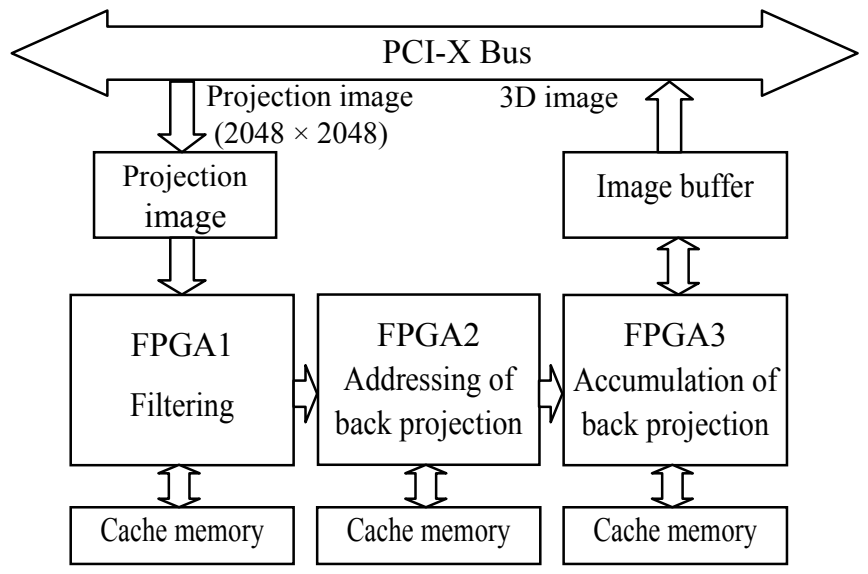

Fig.6 Block diagram of the CT reconstruction acceleration unit. It consists of three FPGAs (field programmable gate arrays) with a fast cache memory. The projection and 3D image are transferred over the PCI-X slot.

\section{EXPERIMENTAL RESULT AND DISCUSSION}

OCT was applied to the analysis of a BGA mounted board. An evaluation board, on which a plastic BGA with a bump pitch of $1.0 \mathrm{~mm}$ and bump diameter of $0.6 \mathrm{~mm}$ was mounted were employed. The PCB size was $150 \times 150 \mathrm{~mm}^{2}$ and the thickness was $2.5 \mathrm{~mm}$. If we had used the conventional vertical CT, we would have had to trim away sections of the PCB except for the analysis region. On the other hand, we did not have to cut any region of the PCB when using the OCT system.

Table I shows the experimental conditions. Fig. 7 shows the horizontal slice image, and Fig. 8 shows the vertical slice image of OCT. In Fig.7 (b), the bump outline at the defective bump is unclear, which indicates that the solder is not connected correctly between the bump and the PCB. Additionally, Fig. 8 also indicates that some bumps are incorrect at the PCB side.

Regarding to the inspection speed, projection images were captured at the theoretical limitative speed of FPD, and the reconstruction ended under capturing. As a result, scanning was finished in $32 \mathrm{~s}$. When cycle time of production line is 150 s per board, this system allows inspecting four BGA packages without making production line waiting. Our system was 17 times faster than conventional system even if reconstruction time is not concerned.

TABLE I

EXPERIMENTAL CONDITIONS

\begin{tabular}{c|c}
\hline X-ray voltage $[\mathrm{kv}]$ & 100 \\
\hline X-ray current $[\mathrm{uA}]$ & 100 \\
\hline Matrix size of projection image $(\mathrm{X} \times \mathrm{Y})$ & $2048 \times 2048$ \\
\hline Matrix size of OCT image $(\mathrm{X} \times \mathrm{Y} \times \mathrm{Z})$ & $2048 \times 2048 \times 64$ \\
\hline Geometric magnification & 10 \\
\hline Spatial resolution $(\mathrm{X} \times \mathrm{Y} \times \mathrm{Z})[\mu \mathrm{m}]$ & $5.0 \times 5.0 \times 10.0$ \\
\hline Number of projections & 32 \\
\hline Inspection time $[$ sec $/ \mathrm{scan}]$ & 32 \\
\hline
\end{tabular}

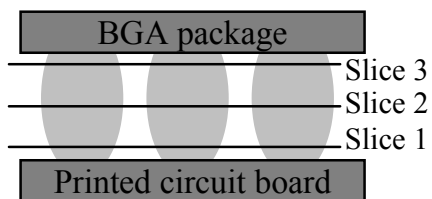

(a)

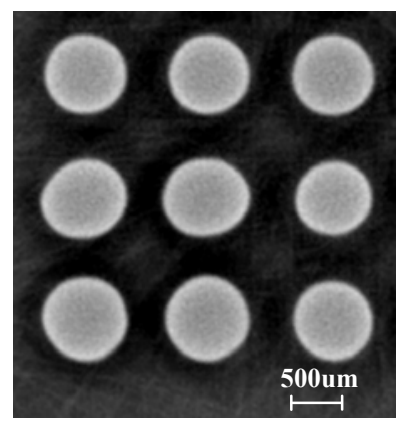

(c)

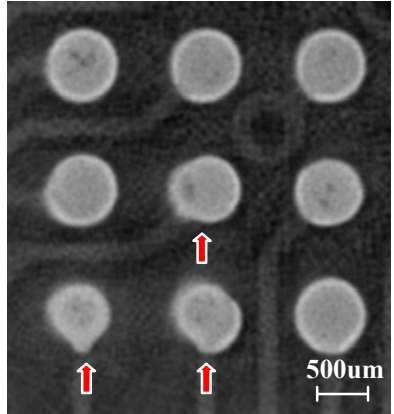

(b)

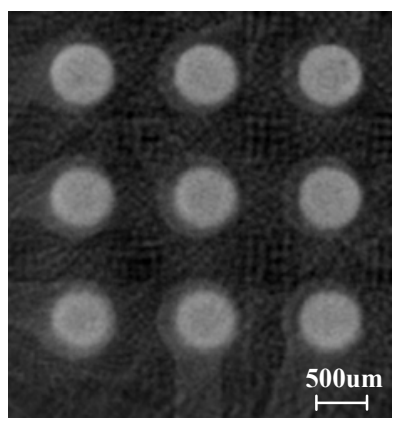

(d)
Fig.7 Horizontal slice images of the BGA-mounted substrate. (a) illustrates the cross-sectional view of the BGA mounted board and the slice position. (b) to (d) show the horizontal slice image at slice 1 to 3 , respectively. Arrows indicate the soldering defect. 


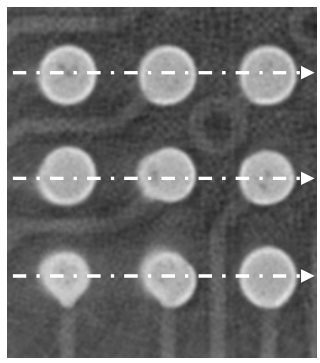

(a)

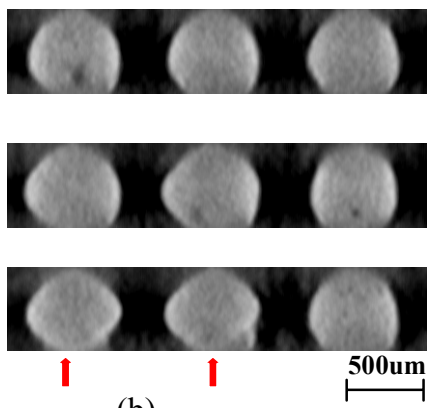

(b)
[9] C.U.Petersson, P.Edholm, G.H.Granlund, and H.E.Knutsson, "Ectomography-A New Radiographic Reconstruction Method-II. Computer Simulated Experiments", IEEE Transactions on Biomedical Engineering, Vol.27, No.11, pp.649-655, 1980.

[10] H.Matsuo, A.Iwata, I.Hoiba, N.Suzumura, "Three-Dimensional Image Reconstruction by Digital Tomo-Synthesis Using Inverse Filtering", IEEE Transactions on Medical Imaging, Vol.12, No.2, pp.307-313, 1993.

Fig.8 Vertical slice images of the BGA-mounted substrate.

(a) horizontal slice image which gives the slice line for the vertical slice (white arrows). (b) vertical slice images generated from the 3D OCT image along the white lines in (a). Arrows indicate the soldering defect.

\section{CONCLUSION}

In this paper, we proposed an oblique CT system that can non-destructively obtain the 3D solder shape at a high speed. This system collects the projection images from various directions using a rotational FPD and a fixed mounted opentype $\mathrm{X}$-ray generator that has a wide radiation angle, and obtains the 3D CT image by the 3D FPB method. In addition, we developed a $\mathrm{CT}$ reconstruction acceleration unit for our $\mathrm{CT}$ system, and the 3D CT image was obtained in real time. In the experiments, we analyzed the BGA mounted board. The results demonstrated that the proposed system was able to obtain the effective information by non-destructive inspection of the BGA mounted PCB accurately.

This system is the world's first CT system, which can be introduced into the soldering line, and it is operated by more than 20 domestic and foreign factories now.

\section{REFERENCES}

[1] P.Haglund, P.Frisk, J.O.Andersson, "BGA-MCM technology for harsh environmental applications", ISHM-Europe, pp.14-16, 1997.

[2] R.Ghaffarian, "BGAs for high reliability applications", Electronics Packaging \& Production, Vol. 38, No.3, pp.45-52, 1998.

[3] A.Teramoto, I.Horiba, "Extraction of Solder Constituent from CSP Boards by Means of X-ray Energy Subtraction Method", Journal of Japan Institute of Electronics Packaging, Vol.4, No.1,pp.68-71, 2001, in Japanese.

[4] A.Teramoto, T.Murakoshi, I.Horiba, "High Speed and High Precision Solder Bump Inspection Method Using X-ray Fluoroscopy", IEEJ Transactions on Industry Applications, Vol.125-D,No.6, pp.652-658 , 2005, in Japanese.

[5] A.Teramoto, T.Murakoshi, M.Tsuzaka, H.Fujita, "Automated Detection of Micro Void in Solder Bump", IEEJ Transactions on Industry Applications, Vol.126, No.11, pp.1514-1421, 2006, in Japanese.

[6] L.A.Shepp and J.B.Kruskal, "Computerized tomography: The new medical X-ray technology", American Mathematical Monthly, vol.85, pp.655-661, 1979.

[7] Z.H.Cho, "General views on 3-D Image Reconstruction and Computed Transverse Axial Tomography", IEEE Transactions on Nuclear Science, vol.21, pp.44-71, 1974.

[8] H.E.Knutsson, P.Edholm, G. H.Granlund, and C.U.Petersson, "Ectomography-A New Radiographic Reconstruction Method-I. Theory and Error Estimates", IEEE Transactions on Biomedical Engineering, Vol.27, No.11, pp.640-648, 1980. 